\title{
Experimental comparison of torque control methods on an ankle exoskeleton during human walking
}

\author{
Juanjuan Zhang ${ }^{1,2, *}$, Chien Chern Cheah $^{2}$, Steven H. Collins ${ }^{1,3}$,
}

\begin{abstract}
Few comparisons have been performed across torque controllers for exoskeletons, and differences among devices have made interpretation difficult. In this study, we compared the torque-tracking performance of nine control methods, including variations on classical feedback control, modelbased control, adaptive control and iterative learning. Each was tested with four high-level controllers that determined desired torque based on time, joint angle, a neuromuscular model, or electromyography. Controllers were implemented on a tethered ankle exoskeleton with series elastic actuation. Measurements were taken while one human subject walked on a treadmill at $1.25 \mathrm{~m} \cdot \mathrm{s}^{-1}$ for one hundred steady-state steps. The combination of proportional derivative control with iterative learning resulted in the lowest errors for all high-level controllers. With timebased desired torque, rms errors were $0.6 \mathrm{~N} \cdot \mathrm{m}(1.3 \%$ of peak torque) step by step, and $0.1 \mathrm{~N} \cdot \mathrm{m}(0.2 \%)$ on average. These results indicate that model-free, integration-free feedback control is suited to the uncertain dynamics of the human-robot system, while iterative learning is effective in the cyclic task of walking.

Index Terms-Rehabilitation Robotics, Ankle Exoskeleton, Torque Control, Human-Robot Interaction
\end{abstract}

\section{INTRODUCTION}

Exoskeletons have been used for performance restoration [1] and enhancement [2]. Recently, the importance of the natural dynamics of the human body [3], energy input [4] and comfort of human-robot interactions [5-7] has been given increased attention in exoskeleton applications. In these approaches to exoskeleton assistance, torque control is crucial for performance. In such systems, series-elastic actuators [8] are commonly used to provide low-error torque tracking to deal with the unknown and changing human dynamics.

It has been a common interest for the lower-limb exoskeleton community to improve the locomotion performance. The ankle joint has drawn major attention for effort reduction in walking [9] since it produces most of the mechanical work [10]. Better ankle joint torque tracking would therefore greatly benefit experimental studies. Such techniques are also expected be extendable to knee and hip exoskeletons, for which the control problem is similar.

This material is based upon work supported by the National Science Foundation under Grant No. IIS-1355716 and by the Singapore Economic Development Board NTU-CMU Dual PhD Scholarship.

${ }^{1}$ Dept. Mechanical Engineering, Carnegie Mellon University, USA.

${ }^{2}$ School of Electric and Electronic Engineering, Nanyang Technological University, Singapore.

${ }^{3}$ Robotics Institute, Carnegie Mellon University, USA.

* Corresponding author: J. Zhang. Dept. Mechanical Engineering, Carnegie Mellon University, 5000 Forbes Ave. Pittsburgh, Pennsylvania 15213, USA. Email:juanjuaz@andrew.cmu.edu
Control of exoskeletons is normally hierarchical with high level controllers determining behavior-related desire torques and torque control lying at low-level. Therefore, the torque controllers will be referred to as low-level controllers and the desired torque generators as high-level controllers in this study.

Many low-level control methods have been employed for torque or position tracking in exoskeletons, including classical feedback control [7], model-based control [6], adaptive control [11] and iterative learning control [12]. However, it remains unclear which method has the best performance, or how performance may vary with high-level controllers.

High-level controllers based on time [4], joint angle [13], neuromuscular models [7], and electromyographic measurements [9] have been used to assist human walking. Each may be advantageous in some assistance paradigm, and each generates desired torques with different dynamics.

The topic of exoskeleton torque control has not drawn significant attention compared to high level control and biomechanics experiments. In cases where torque control were addressed directly, it has typically been investigated under unrealistic conditions, i.e., during benchtop tests rather than human-robot interactions [14], and results have often not been reported quantitatively [15]. Moreover, no systematic comparisons of torque control methods have been made on the same platform, making differentiation among candidate methods very difficult. This study aims to compare the torquetracking performance of prominent torque controller, under realistic experimental conditions, with multiple high-level controllers, in a single exoskeleton platform. These results are expected to help guide the selection and tuning of exoskeleton torque controller, particularly in lower-limb exoskeletons for locomotion assistance.

\section{Methods}

Nine torque controllers, including variations and combinations of classical feedback control, model-based control, adaptive control and iterative learning, were compared by experiments in this study. Each was tested seperately with four high-level controllers that determined desired torque based on time, ankle angle, a neuromuscular model, or electromyographic measurements. Controllers were implemented on a tethered ankle exoskeleton with series-elastic actuation and tuned to minimize error. Under each high- and low-level controller combination, the exoskeleton was tested with one subject who walked one hundred steady steps on a treadmill. The root mean squared torque errors were calculated for each step and for an averaged step. 


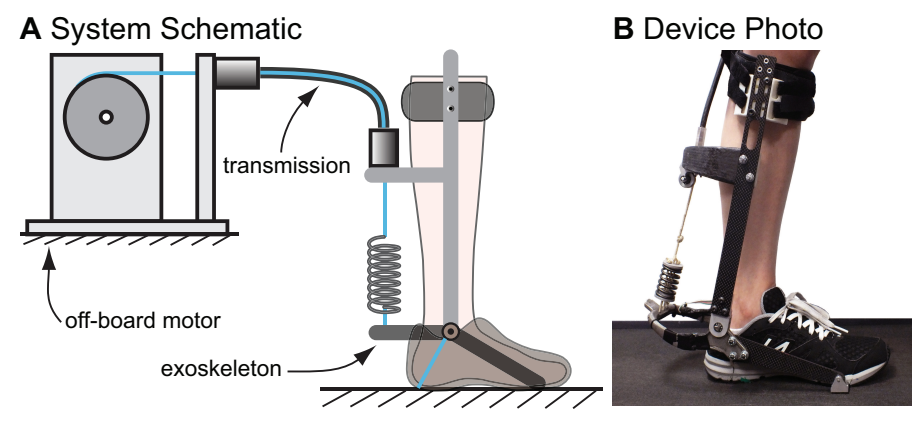

Fig. 1: Schematic and photo of the tethered ankle exoskeleton system. (A): Schematic of the system that consists of an off-board motor, a Bowden cable transmission and an exoskeleton frame; (B): Photo of the exoskeleton worn by a human.

A detailed description of the methodology and complete results for this study are available in a paper submitted to International Journal of Robotics Research [16].

\section{A. Exoskeleton Testbed}

The ankle exoskeleton testbed for experiments consisted of an off-board geared electric motor with real-time driver, a flexible Bowden cable transmission with series compliance, and an exoskeleton that interfaced with the human body (Fig. 1).

A dedicated real-time control system sampled sensor data at $5000 \mathrm{~Hz}$, which were then filtered at $200 \mathrm{~Hz}$. The desired motor velocity commands were generated at $500 \mathrm{~Hz}$. The motor unit was composed of a low-inertia $1.6 \mathrm{~kW}$ AC servo motor and a 5:1 planetary gear, with input voltage regulated by a motor driver running in velocity control mode. A digital optical encoder measured motor position.

The flexible Bowden cable that transmitted forces composed of a coiled-steel outer conduit and a $0.003 \mathrm{~m}$ diameter Vectran ${ }^{\circledR}$ inner rope, and was $2 \mathrm{~m}$ in length. A coil spring with an effective stiffness of $190 \mathrm{~N} \cdot \mathrm{m} \cdot \mathrm{rad}^{-1}$ was attached at the end of the rope to provide increased compliance.

The exoskeleton frame generated a plantarflexion torque which is measured using strain gauges on the heel lever with $1000 \mathrm{~Hz}$ signal conditioning. Joint angle was measured using a digital optical encoder. Muscle activity was measured using a wired electromyography system for one high-level controller.

Various components of the system interacted as in Fig. 2. The high-level controller used time, $t$, exoskeleton joint angle, $\theta_{a}$, or electromyography (EMG) to determine the desired torque, $\tau_{\text {des }}$. The low-level controller tracked torque by changing the desired motor velocity, $\dot{\theta}_{m, d e s}$ determined by desired torque, measured torque, $\tau$, motor angle, $\theta_{m}$, and/or exoskeleton angle. A motor driver regulated motor velocity. Motor motion was transmitted through the Bowden cable to one end of a series spring and generated exoskeleton torque together with exoskeleton motion. Both the human and the series spring exerted torques on the exoskeleton frame and led to exoskeleton rotation.

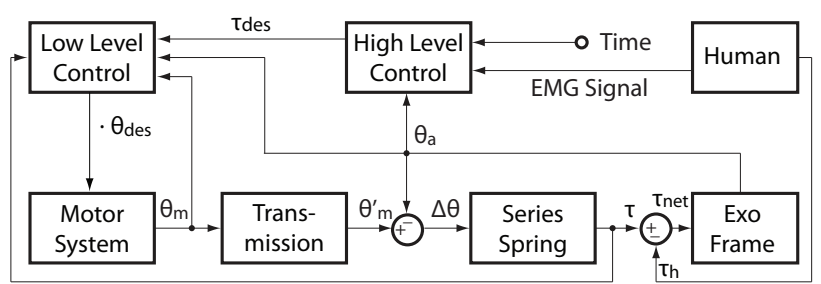

Fig. 2: Flowchart of the control system.

\section{B. Controllers}

Nine prominent low-level torque control methods were compared in this study in combination with four high-level assistance controllers. High-level controllers generated desired torque, which low-level controllers tracked.

1) Low-Level Torque Controllers: Desired motor velocity was commanded to a dedicated hardware motor controller for all torque controllers investigated:

$$
\dot{\theta}_{m, d e s}=\Delta \theta_{m, d e s} T^{-1}=\left[\theta_{m, d e s}-\theta_{m}\right] T^{-1}
$$

where $\dot{\theta}_{m, d e s}$ is the commanded motor velocity; $T$ is a constant related to motor position rise time; $\Delta \theta_{m, d e s}$ is the desired motor displacement; $\theta_{m, \text { des }}$ is the desired motor position; $\theta_{m}$ is the measured motor position.

${ }_{L}$ 1: Proportional Control with Damping Injection (PD)

This controller was a variant of the classical proportionalderivative control of torque, with the derivative term replaced by damping injection [17]:

$$
\Delta \theta_{m, d e s}=-K_{p} e_{\tau}-K_{d} \dot{\theta}_{m}
$$

where $K_{p}$ is proportional gain, $e_{\tau}=\tau-\tau_{\text {des }}$ is torque error, $\tau$ is measured exoskeleton torque, $\tau_{d e s}$ is desired exoskeleton torque, $K_{d}$ is damping gain, and $\dot{\theta}_{m}$ is measured motor velocity. Lower noise was obtained with damping injection compared to the derivative of torque error since motor position was measured by a digital encoder while torque was measured by analog strain gauges.

${ }_{L} 2$ : Proportional Control with Damping Injection and ErrorDependent Gains (PD+EDG)

This controller was the same as ${ }_{L} 1$, except that the proportional gain was error-dependent [18]:

$$
\begin{aligned}
k_{p} & =\min \left[\operatorname{ceil}\left(\frac{\left|e_{\tau}\right|}{h_{\tau}}\right) h_{k}, K_{\max }\right] \\
\Delta \theta_{m, d e s} & =-k_{p} e_{\tau}-K_{d} \dot{\theta}_{m}
\end{aligned}
$$

where operation 'ceil' rounds the element to the next smallest integer, $k_{p}$ is the error-dependent proportional gain, $h_{\tau}$ and $h_{k}$ are torque error and proportional gain step sizes, and $K_{\max }$ is the maximum allowable gain. This controller was intended to limit overshoot and oscillations during torque tracking.

$L^{3}$ : Proportional Control with Damping Injection and Previous-Error Compensation (PD+PEC)

This controller was the same as ${ }_{L} 1$, except that desired torque was altered based on torque error from the previous time step [19]:

$$
\begin{aligned}
\tau_{\text {des }}^{\prime} & =\tau_{\text {des }}-e_{\tau, \text { prev }} \\
\Delta \theta_{m, \text { des }} & =-K_{\text {pec }}\left(\tau-\tau_{\text {des }}^{\prime}\right)-K_{d} \dot{\theta}_{m}
\end{aligned}
$$


where $\tau_{\text {des }}^{\prime}$ is the compensated desired torque and $e_{\tau, \text { prev }}$ is the torque error from the previous time step. This was intended to increase control response for large errors.

${ }_{L}^{4}$ : Proportional-Integral Control with Damping Injection (PID)

This controller was a variant of the classical proportionalintegral-derivative control, with the derivative term replaced by damping injection:

$$
\Delta \theta_{m, d e s}=-K_{p} e_{\tau}-K_{i} \int_{t_{0}}^{t} e_{\tau} d t-K_{d} \dot{\theta}_{m}
$$

where $K_{i}$ is the gain on the integral of torque error, and $t_{0}$ is the time at which the step began.

${ }_{L}$ 5: Proportional Control with Damping Injection and Modelbased Compensation (PD+M)

This controller combined the feedback controller of ${ }_{L} 1$ and a model-based feed-forward term, which was intended to anticipate changes in desired motor position due to either exoskeleton joint displacements or changes in desired joint torque:

$$
\begin{aligned}
\Delta \theta_{m, d e s} & =-K_{p} e_{\tau}-K_{d} \dot{\theta}_{m}+\left(\theta_{m d l}-\theta_{m}\right) \\
\theta_{m d l} & =\theta_{a} \tilde{R}-\tau_{d e s} \tilde{K}_{c}^{-1}
\end{aligned}
$$

where $\theta_{m d l}$ is model-based motor position compensation, $\theta_{m}$ is measured motor position, $\theta_{a}$ is measured exoskeleton ankle joint angle, $\tilde{R}$ is estimated total gear ratio from motor to exoskeleton joint, and $\tilde{K}_{c}$ is estimated total stiffness of the transmission with respect to the motor displacement.

${ }_{L}$ 6: Passivity-based Adaptive Control (PAS)

This provably stable adaptive controller was based on a dynamic model of the motor, transmission and exoskeleton. It is described as

$$
\Delta \theta_{m, d e s}=-K_{p} e_{\tau}-K_{s} s+Y_{d}\left(\tau, \dot{\tau}_{r}, \ddot{\tau}_{r}, \dot{\theta}_{a}\right) \hat{\Gamma}
$$

where $K_{s}$ is the sliding control gain and $s$ is a sliding vector defined as

$$
s=\dot{\tau}-\dot{\tau}_{\text {des }}+\lambda e_{\tau}=\dot{\tau}-\dot{\tau}_{r}
$$

where $\lambda$ is a positive scalar and $\tau_{r}$ is a virtual reference torque. $Y_{d}$ is a regressor defined as

$$
Y_{d}\left(\tau, \dot{\tau}_{r}, \ddot{\tau}_{r}, \dot{\theta}_{a}\right)=\left[\tau \dot{\tau}_{r} \ddot{\tau}_{r} \dot{\theta}_{a}\right]
$$

which expresses the dynamics as a linear combination of system parameters. $\Gamma$ is the system parameter vector. Its estimate $\hat{\Gamma}$ was updated by the law

$$
\dot{\hat{\Gamma}}=-L Y_{d}^{T} s
$$

where $\mathrm{L}$ is a positive definite parameter adaptation gain matrix.

This controller was intended to reduce model uncertainties by parameter adaptation and address unknown human-robot interactions.

${ }_{L} 7$ : Iterative Learning of Desired Motor Position (LRN)

This controller continuously updated a desired motor position trajectory for the next step using torque errors of the current step [12]:

$$
\begin{aligned}
\theta_{m, \text { des }}(i, n+1) & =\beta \theta_{m, \text { des }}(i, n)-K_{l} e_{f l t}(i, n) \\
\Delta \theta_{m, \text { des }}(i, n) & =\theta_{m, \text { des }}(i+D, n)-\theta_{m}(i, n)
\end{aligned}
$$

where $i$ is the time index or number of control cycles elapsed within this step, $n$ is this step and $n+1$ is the next step, $K_{l}$ is the iterative learning gain, and $D$ is an estimate of the delay between commanding and achieving a change in motor position. Current torque error thereby updates desired motor position for the same time index on the next step, while commanded motor velocity at this time index is based on a preview of desired motor position later in the same step.

$\beta \in[0,1]$ is a weighting term on the learned trajectory to add "forgetting" into learning. $e_{f l t}$ is the filtered torque error trajectory, initially an array of zeros, expressed as

$$
e_{f l t}(i, n)=(1-\mu) e_{f l t}(i, n-1)+\mu e_{\tau}(i, n)
$$

where $\mu \in[0,1]$ is a weighting term on the learned error. This controller exploited the cyclic nature of the task to accommodate complex system dynamics without an explicit model .

${ }_{L}$ 8: Iterative Learning of Desired Motor Position + Proportional-Damping Compensation (LRN+PD)

This controller combined iterative learning $\left({ }_{L} 7\right)$ with proportional-damping control $\left.{ }_{L}{ }_{L} 1\right)$ compensation. Motor commands arose primarily from the learned feed-forward trajectory, while feedback control compensated for stepby-step variations in desired torque:

$$
\begin{aligned}
\theta_{m, d e s}^{L R N}(i, n+1)= & \beta \theta_{m, d e s}^{L R N}(i, n)-K_{l} e_{f l t}(i, n) \\
\theta_{m, d e s}(i, n)= & \theta_{m, d e s}^{L R N}(i+D, n) \\
& -K_{p} e_{\tau}(i, n)-K_{d} \dot{\theta}_{m}(i, n) \\
\Delta \theta_{m, d e s}(i, n)= & \theta_{m, d e s}(i, n)-\theta_{m}(i, n)
\end{aligned}
$$

${ }_{L}$ 9: Proportional Control with Damping Injection + Iterative Learning Compensation (PD+LRN)

This controller combined proportional-damping control $\left({ }_{L} 1\right)$ with iterative learning $\left({ }_{L} 7\right)$ compensation. Motor commands arose primarily from feedback control, while a learned feed-forward component compensated for stepwise consistent tracking errors:

$$
\begin{aligned}
\Delta \theta_{m, d e s}^{L R N}(i, n+1)= & \beta \Delta \theta_{m, d e s}^{L R N}(i, n)-K_{l} e_{f l t}(i, n) \\
\Delta \theta_{m, d e s}(i, n)= & -K_{p} e_{\tau}(i, n)-K_{d} \dot{\theta}_{m}(i, n) \\
& +\Delta \theta_{m, d e s}^{L R N}(i+D, n)
\end{aligned}
$$

2) High-Level Assistance Controllers: During the stance phase, desired torque $\tau_{\text {des }}$ was determined by high-level controllers $H^{1-} H^{4}$ detailed below. During the swing phase, motor position control was employed to allow free human ankle movement motion while maintaining minimal cable slack:

$$
\Delta \theta_{m, \text { des }}=\theta_{a} \tilde{R}-\theta_{m}
$$

where $\tilde{R}$ is the estimated total gear ratio from motor to exoskeleton joint.

\section{${ }_{H}$ 1: Time Based Desired Torque Trajectory (TIME)}

This high-level controller set desired torque as a function of time. We used a curve (Fig. 3) that resembled a scaled-down version of the human ankle moment during unassisted walking [10] with a stance period of $0.66 \mathrm{~s}$.

$H^{2}$ : Joint Angle Based Desired Torque (ANGLE)

This high-level controller set desired torque as a function of ankle angle and phase of the gait cycle [13]. We used a piece-wise linear curve that resembled a scaled-down version of the human ankle moment during unassisted walking (Fig. 4), calculated as $\tau_{d e s}=\frac{\tau_{i}-\tau_{i-1}}{\theta_{a, i}-\theta_{a, i-1}}\left(\theta_{a}-\theta_{a, i-1}\right)$ 


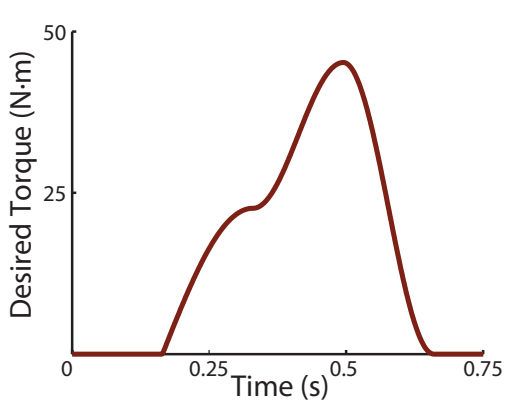

Fig. 3: High-level control based on a trajectory in time

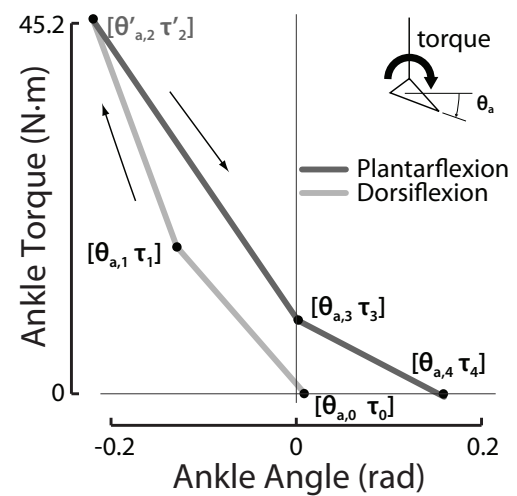

Fig. 4: High-level control based on ankle joint angle

$(i=1,2,3,4)$ in which $\left(\theta_{i}, \tau_{i}\right)$ defines a node in torqueangle space. The node $\left(\theta_{2}, \tau_{2}\right)$ marked the transition from the Dorsiflexion phase, in which ankle velocity was negative, to the Plantarflexion phase, in which ankle velocity was positive. Since the exact transition point varied on each step, the angle and torque at the moment of transition, $\left(\theta_{2}^{\prime}, \tau_{2}^{\prime}\right)$ replaced $\left(\theta_{2}, \tau_{2}\right)$ for calculating desired torque in the first portion of Plantarflexion, i.e., when $i=3$.

$H^{3}$ : Neuromuscular Model Based Desired Torque (NMM) This high-level controller set desired torque using a virtual muscle and neural system model. The virtual muscle took human ankle position and velocity as inputs and generated a virtual joint torque which was conditioned and used as desired torque. The virtual torque generated was also fed into the virtual neural system and linearly scaled to generate the stimulation signal of the virtual muscle. This virtual reflex mechanism realized a form of positive feedback in the model. A complete description of the reflex-based muscle model was available in [7] and the details of the implementation of the model in this study is available in [16].

$H^{4}$ : Electromyography Based Desired Torque (EMG)

This high-level controller set desired torque in proportion to EMG measurements [9] from subject's gastrocnemius muscle. Electrical activity was measured using surface electrodes, high-pass filtered at $20 \mathrm{~Hz}$, rectified, low-pass filtered at $6 \mathrm{~Hz}$, offset by a small value of -0.008 , and amplified by a gain of 283 to obtain the desired torque.

\section{Experimental Methods}

Experiments were conducted with one healthy subject (30 yrs, $56 \mathrm{~kg}, 1.65 \mathrm{~m}$ tall, female), who walked on a treadmill at $1.25 \mathrm{~m} \cdot \mathrm{s}^{-1}$ with a self-paced step period of $1.08 \pm 0.06 \mathrm{~s}$ while wearing the exoskeleton on the right leg.

Before collecting data, we tuned parameters for each combination of high- and low-level controller as the subject walked with the exoskeleton. High-level control parameters for ${ }^{1}{ }^{1-} H^{4}$ were selected so as to result in peak desired torques of approximately $45 \mathrm{~N} \cdot \mathrm{m}$. Low-level control parameters listed in Table I were systematically tuned with the aim of minimizing torque error. During tuning, very similar optimal low-level control parameters across high-level controllers were seen. So, same values were used for high-level controllers. For model-based compensation, the value of $\tilde{R}$ was based on measurements. $\tilde{K}_{c}$ was determined based on measured lever arm and the reported stiffness of the series spring.

TABLE I: Low-level torque control parameter values

\begin{tabular}{l|l||c|l||c|l}
\hline Param. & Value & Param. & Value & Param. & Value \\
\hline$K_{p}$ & 0.093 & $\tilde{R}$ & 2.90 & $K_{L}$ & 0.0077 \\
$K_{d}$ & 0.010 & $\tilde{K}_{c}$ & $195 \mathrm{~N} \cdot \mathrm{m} \cdot \mathrm{rad}^{-1}$ & $D$ & $0.022 \mathrm{~s}$ \\
$K_{\max }$ & 0.15 & $K_{s}$ & 0.005 & $\beta$ & 1 \\
$K_{\text {pec }}$ & 0.046 & $\lambda$ & 0.077 & $\mu$ & 1 \\
$K_{i}$ & $7.7 \mathrm{e}-5$ & $L$ & $1.0 \mathrm{e}-9$ & $T$ & $0.050 \mathrm{~s}$ \\
$h_{\tau}$ & $11.3 \mathrm{~N} \cdot \mathrm{m}$ & $h_{k}$ & 0.039 & & \\
\hline
\end{tabular}

For each high-level condition, all low-level controllers were tested on the same day, without removal of the exoskeleton between trials. For each combination of low- and highlevel controllers, data of one hundred steady-state steps were collected. Root mean squared torque error both for the set of all steady-state steps and for the average step, which was obtained by averaging the desired and actual trajectories of the one hundred steps in time, were then calculated.

\section{RESULTS}

The best torque tracking performance was observed with the combination of feedback control and iterative learning, i.e., PD+LRN or LRN+PD, for all high-level controllers, both in real-time and for the average step. Between the two, PD+LRN showed lower errors before convergence. Depending on highlevel conditions, the real-time torque errors with PD+LRN were $38 \%-84 \%$ lower than with PD (t-test $p<1.9 \mathrm{e}-34$ ), and average-step torque errors were $91 \%-97 \%$ lower. Other additions to feedback control showed minor performance effects, except that model-based compensation increased torque error. While providing low errors for average tracking, pure iterative learning saw high real-time errors. For the EMGbased condition, torque tracking errors and variability were generally higher for all torque controllers.

Some interactions between high- and low-level torque controllers were observed. Pure feedback control outperformed pure iterative learning control for Angle and EMG based highlevel conditions, while an opposite trend was seen in Time and NMM. Under Time-based condition, poor tracking at the onset of desired torque, including a delay and overshoot, was 


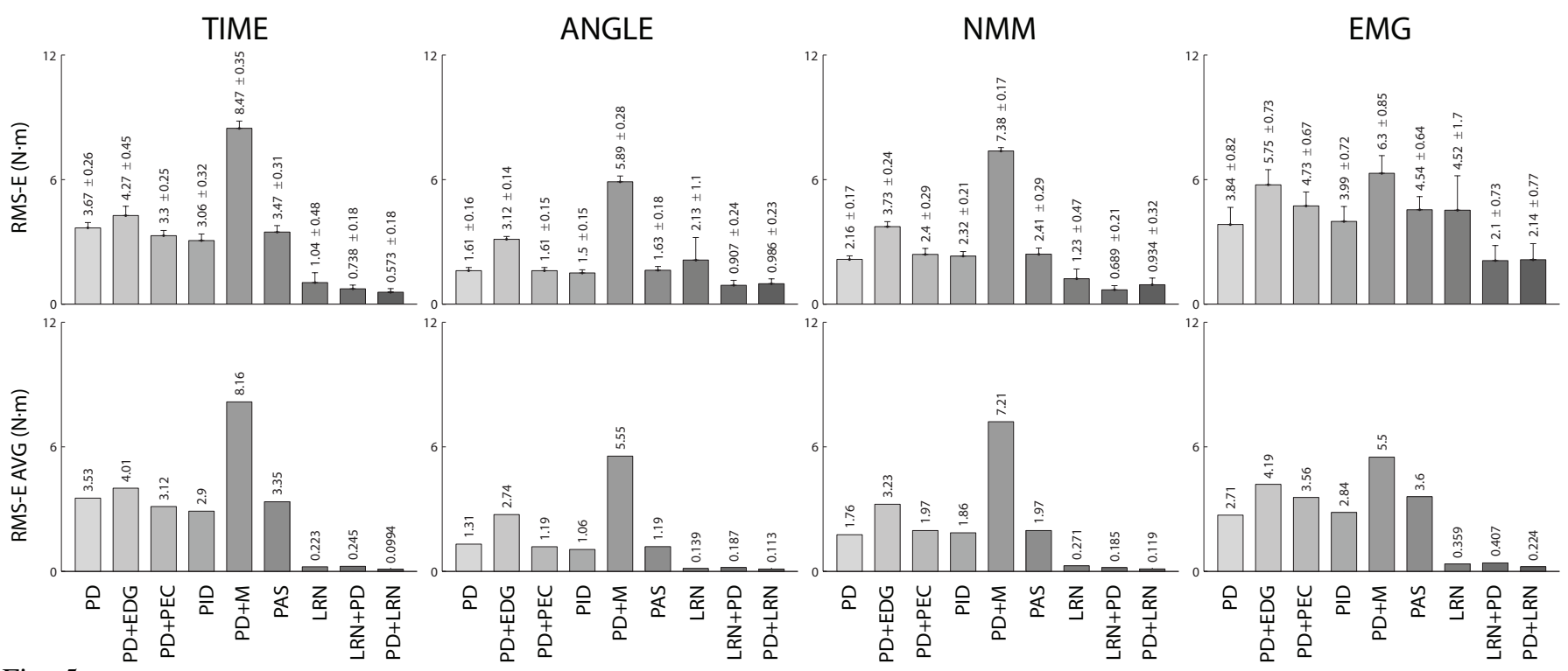

Fig. 5: Root-mean-squared torque error calculated for all steps (RMS-E) and for an average step (RMS-E AVG) across all high- and low-level control combinations.

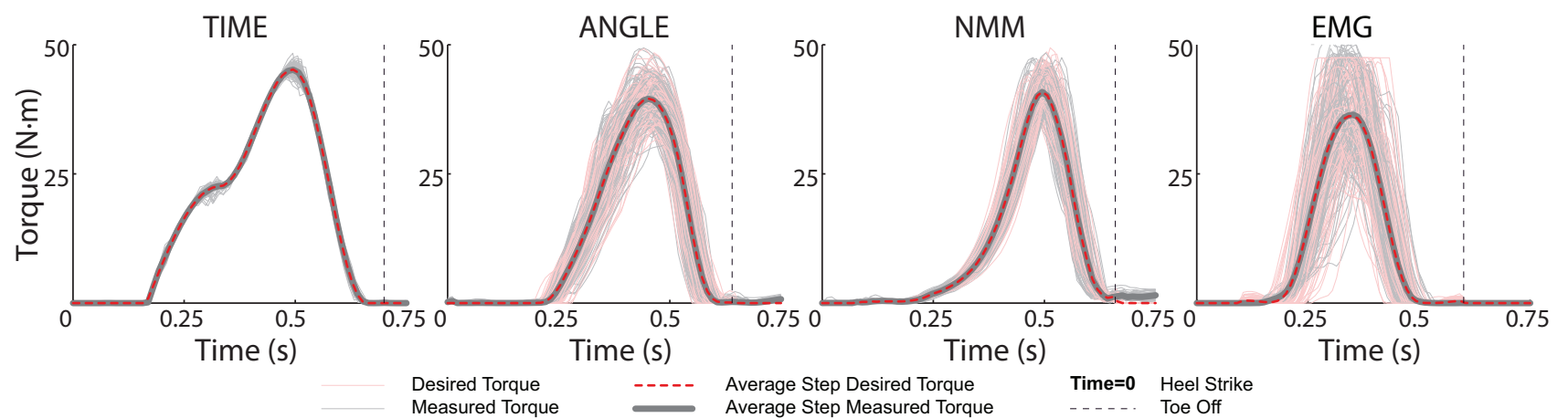

Fig. 6: Overlapped desired and measured torques in time space for all high level controllers with proportional control with damping injection compensated by iterative learning $(\mathrm{PD}+\mathrm{LRN})$

observed for all controllers without a learning component. Adding iterative learning to PD resulted in the greatest improvement in performance for Time based condition. Minor improvement in performance over pure PD was seen with an additional integral term (PID) for Time and Angle based conditions. For Time-based high-level control, passivity (PAS) and previous-error compensation $(\mathrm{PD}+\mathrm{PEC})$ also showed a small benefit.

Means and standard deviations of step-wise root-meansquared torque error (RMS-E) and average-step root-meansquared error (RMS-E AVG) are shown in Fig. 5. Error values for PD+LRN and their percentage of the peak desired torque for the average step, are given in Table II. Overlapped desired and measured torque trajectories for one hundred steps with PD+LRN are shown in Fig. 6. Real-time tracking performance of this controller are demonstrated by four consecutive steps at steady state in Fig. 8 of Appendix B. PD+LRN with Anglebased desired torque also showed consistent performance for higher torque on two different devices (peak torques $86.3 \pm 8.5 \mathrm{~N} \cdot \mathrm{m}$ and $81.2 \pm 7.7 \mathrm{~N} \cdot \mathrm{m}$ respectively) with a taller male subject in later experiments (Fig. 7 in Appendix A).
TABLE II: Tracking errors with PD+LRN torque control

\begin{tabular}{c||c|c||c|c}
\hline & RMS-E & $\% \tau_{\max }$ & RMS-E AVG & $\% \tau_{\max }$ \\
\hline Time & $0.57 \pm 0.18 \mathrm{~N} \cdot \mathrm{m}$ & $1.3 \%$ & $0.10 \mathrm{~N} \cdot \mathrm{m}$ & $0.2 \%$ \\
Angle & $0.99 \pm 0.23 \mathrm{~N} \cdot \mathrm{m}$ & $2.5 \%$ & $0.11 \mathrm{~N} \cdot \mathrm{m}$ & $0.3 \%$ \\
NMM & $0.93 \pm 0.32 \mathrm{~N} \cdot \mathrm{m}$ & $2.3 \%$ & $0.12 \mathrm{~N} \cdot \mathrm{m}$ & $0.3 \%$ \\
EMG & $2.14 \pm 0.77 \mathrm{~N} \cdot \mathrm{m}$ & $5.9 \%$ & $0.22 \mathrm{~N} \cdot \mathrm{m}$ & $0.6 \%$ \\
\hline
\end{tabular}

\section{Discussion}

We evaluated the performance of nine prominent torque controllers by experiments on a tethered ankle exoskeleton, with series-elastic actuation, during human walking, with four of high-level controllers. Model-free proportional control with damping injection, compensated by iterative learning, resulted in the lowest torque errors for all high-level controllers, both in real-time and for the average step. This approach is analogous to the classical proportional-integral-derivative control; the proportional term provides basic tracking, iterative learning eliminates steady-state cyclic errors, and damping injection provides stability. We thus label the method as proportionallearning-damping control.

Little attention has been paid to torque control in the field 
of exoskeletons and active orthoses. Few quantitative reports on torque control for lower-limb exoskeletons are available, which were mostly benchtop tests [14]. These tests usually do not reflect the unknown, complex and time-varying dynamics of human-robot interactions, which define the biggest challenge in exoskeleton torque control. It is also the case that little data and analysis have been provided [15], which makes interpretation of the results difficult. From the information available, proportional-learning-damping control demonstrated here showed substantially better performance than existing approaches.

Some interactions between high- and low-level controllers were observed. Iterative learning led to bigger improvement in torque tracking for the Time-based high-level controller, presumably because the desired motor position were also learned in time. Therefore, it might be that for Angle-based desired torque, iterative learning elements could be more effective if they were performed based on joint angle. When PD control was compensated by an integral term (PID), previous-error compensation (PD+PEC), or passivity-based adaptation (PAS), torque error with Time-based high-level control was slightly improved, which suggested that the model-free continuoustime integration elements in torque controllers may be beneficial when the desired torque was consistent in time. Errordependent gains (PD+EDG) did not provide benefits and may be more suited for motion based control in rehabilitation.

While showing promise in simulation and theory, modelbased control elements generally worsened or had no effect on control performance in our experiments. Sensitivity to modeling accuracy seems to be a fundamental issue. The partially model-based PAS controller showed slight benefit due to its adaptive nature, but not substantial.

Comparisons across high-level controllers were difficult to make for the same low-level control due to un-normalized tracking difficulty. Multiple values for high-level parameters were not tested in this study, which is an area for future work.

Changes in the patterns of desired torque and joint kinematics across low level controllers for the same high-level condition reveal an interaction effect. For example, more variability in desired torque with NMM-based than Anglebased assistance was observed using PD, but an opposite trend for LRN control was seen. This seems to be due to the complex, multi-time-scale, dynamic interactions between continuous behavior of the torque controller, within-step human variations, high-level controller, and human adaptation over multiple steps. These effects may be important in selecting and tuning an exoskeleton torque controller.

Hardware, series elasticity in particular, also interacted with the torque control performance. Investigation of interactions between series elasticity, torque controller, high-level controller, and assisted tasks should be part of future work.

\section{Conclusions}

A systematic comparison of exoskeleton torque controller under walking condition was conducted in this study, which showed that proportional control with damping injection compensated by iterative learning had better torque tracking performance than any other methods tested or previously demonstrated. Implementation of this proportional-learning-damping controller was straightforward, requiring sequential tuning of four parameters. Our results support the application of this approach on any torque-controlled exoskeleton used during locomotion. There remains a rich area for future research on complex interactions between exoskeleton hardware, torque control, assistance control, task goals and human behavior.

\section{ACKNOWLEDGEMENT}

The authors thank Rachel Jackson and Kirby Witte for assistance with hardware improvement.

\section{REFERENCES}

[1] W. Cloud, "Man amplifiers: Machines that let you carry a ton," Popular Science, vol. 187, no. 5, pp. 70-73, 1965.

[2] R. S. Mosher et al., Handyman to hardiman. Society of Automotive Engineers, 1967.

[3] T. Mcgeer, "Passive dynamic walking," The international journal of robotics research, 1990.

[4] P. Malcolm, W. Derave, S. Galle, and D. D. Clercq, "A simple exoskeleton that assists plantarflexion can reduce the metabolic cost of human walking," PLOS ONE, vol. 8, 2013.

[5] J. R. Andres and N. Hogan, "Impedance control as a framework for implementing obstacle avoidance in a manipulator," Control of Manufacturing Processes and Robotic Systems, ASME, pp. 243-251, 1983.

[6] H. Kazerooni, "The berkeley lower extremity exoskeleton project," $E x$ perimental Robotics IX, Springer Tracts in Advanced Robotics, vol. 21, pp. 291-301, 2006.

[7] H. Geyer and H. Herr, "A muscle-reflex model that encodes principles of legged mechanics produces human walking dynamics and muscle activities," IEEE Trans Neural Syst Rehabil Eng, vol. 18, pp. 263-273, 2010.

[8] G. A. Pratt and M. M. Williamson, "Series elastic actuators," in Proc. of International Conference on Robotic Systems, 1995, pp. 399-406.

[9] D. P. Ferris, K. E. Gordan, G. S. Sawicki, and A. Peethambaran, "An improved powered anklefoot orthosis using proportional myoelectric control," Gait \& Posture, vol. 23, pp. 425-528, 2006.

[10] D. A. Winter, The biomechanics and motor control of human gait: normal, elderly and pathological, 2nd ed. Waterloo: University of Waterloo Press, 1991.

[11] A. Calanca, L. Capisani, and P. Fiorini, "Robust force control of series elastic actuators," Actuators, vol. 3, pp. 182-204, 2014.

[12] J. Bae and M. Tomizuka, "A gait rehabilitation strategy inspired by an iterative learning algorithm," Mechatronics, vol. 22, pp. 213-221, 2012.

[13] J. M. Caputo and S. H. Collins, "A universal ankle-foot prosthesis emulator for human locomotion experiments," Journal of Biomechanical Engineering, vol. 136, 2014.

[14] K. B. Fite and M. Goldfarb, "Design and energetic characterization of a proportional-injector monopropellant-powered actuator," IEEE/ASME Transactions on Mechatronics, vol. 11, pp. 196-204, 2006.

[15] A. B. Zoss, H. Kazerooni, and A. Chu, "Biomechanical design of the berkeley lower extremity exoskeleton (bleex)," IEEE/ASME Transactions on Mechatronics, vol. 11, no. 2, Apr. 2006.

[16] J. Zhang, C. C. Cheah, and S. H. Collins, "A systematic comparison of nine prominent torque control methods, on a tethered ankle exoskeleton with series elastic actuation, during human walking," International Journal of Robotics Research, 2014, submitted.

[17] M. Takegaki and S. Arimoto, "A new feedback method for dynamic control of manipulators," Trans. ASME; J. Dyn. Syst., Meas., Contr., vol. 102, pp. 119-125, 1981.

[18] J. Zhang, C. C. Cheah, and S. H. Collins, "Stable human-robot interaction control for upper-limb rehabilitation robotics," in Proceedings of IEEE International Conference on Robotics and Automation, 2013, pp. $2201-2206$.

[19] J. Gordon and C. Ghez, "Trajectory control in targeted force impulses: III. compensatory adjustment for initial erros," Experimental Brain Research, vol. 67, pp. 253-267, 1987. 
APPENDIX A

Demonstration of TORque Tracking Performance OF PD+LRN FOR HIGHER TORQUE AND MULTIPLE DEVICES

Later tests of PD+LRN for Angle-based high level controller showed that the proportional-learning-damping control method performs well with higher torques multiple devices (Fig. 7). The average-step peak desired torques and RMS error values are shown in Table III.

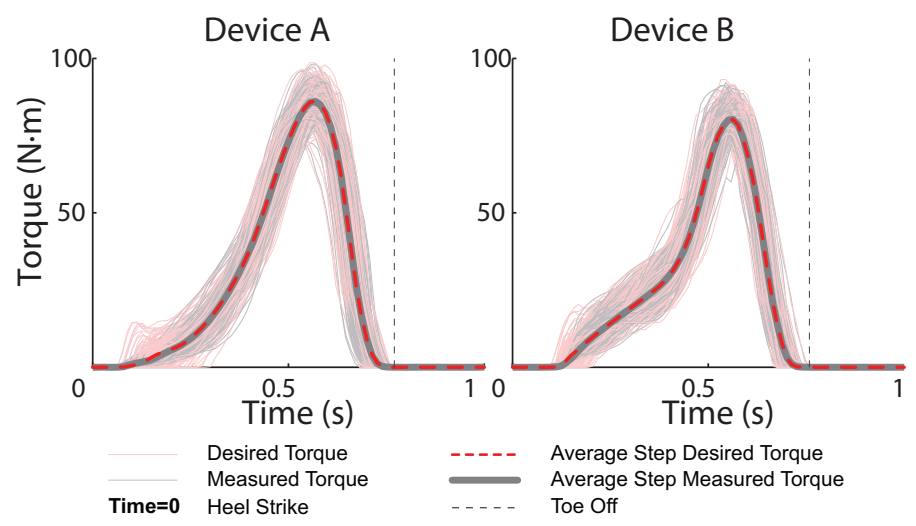

Fig. 7: Torque Tracking Performance of Proportional Control with Damping Injection Compensated by Iterative Learning on higher torque and multiple devices with angle-based high-level controllers. Device A is the one used by the all the comparison experiments, while Device B is another version of the device with similar design concept but leaf spring instead of coil and flexible structure.

TABLE III: Result values of PD+LRN with Angle-based desired torque on higher torque and multiple devices.

\begin{tabular}{l||c|c}
\hline & Device A & Device B \\
\hline \hline Peak Desired Torque & $86.9 \pm 8.5 \mathrm{~N} \cdot \mathrm{m}$ & $81.2 \pm 7.7 \mathrm{~N} \cdot \mathrm{m}$ \\
\hline RMSE & $2.15 \pm 0.55 \mathrm{~N} \cdot \mathrm{m}$ & $1.7 \pm 0.57 \mathrm{~N} \cdot \mathrm{m}$ \\
\hline RMSE \% $\max \left(\tau_{\text {des }}\right)$ & $2.5 \%$ & $2.12 \%$ \\
\hline RMSE AVG & $0.28 \mathrm{~N} \cdot \mathrm{m}$ & $0.24 \mathrm{~N} \cdot \mathrm{m}$ \\
\hline RMSE AVG \% $\max \left(\tau_{\text {des }}\right)$ & $0.32 \%$ & $0.3 \%$ \\
\hline
\end{tabular}

As seen, the performance of PD+LRN with Angle-based desired torque on high torque demonstrated in Table III is very similar to that of low torque as shown in the third row of Table II.

\section{APPENDIX B \\ Demonstration of Torque Tracking Performance OF PD+LRN IN REAL TIME}

To demonstrate the torque tracking performance of Proportional Control with Damping Injection Compensated by Iterative Learning (PD+LRN) in real-time, four-step examples of the torque control methods on different high-level controllers are given in Fig. 8. 

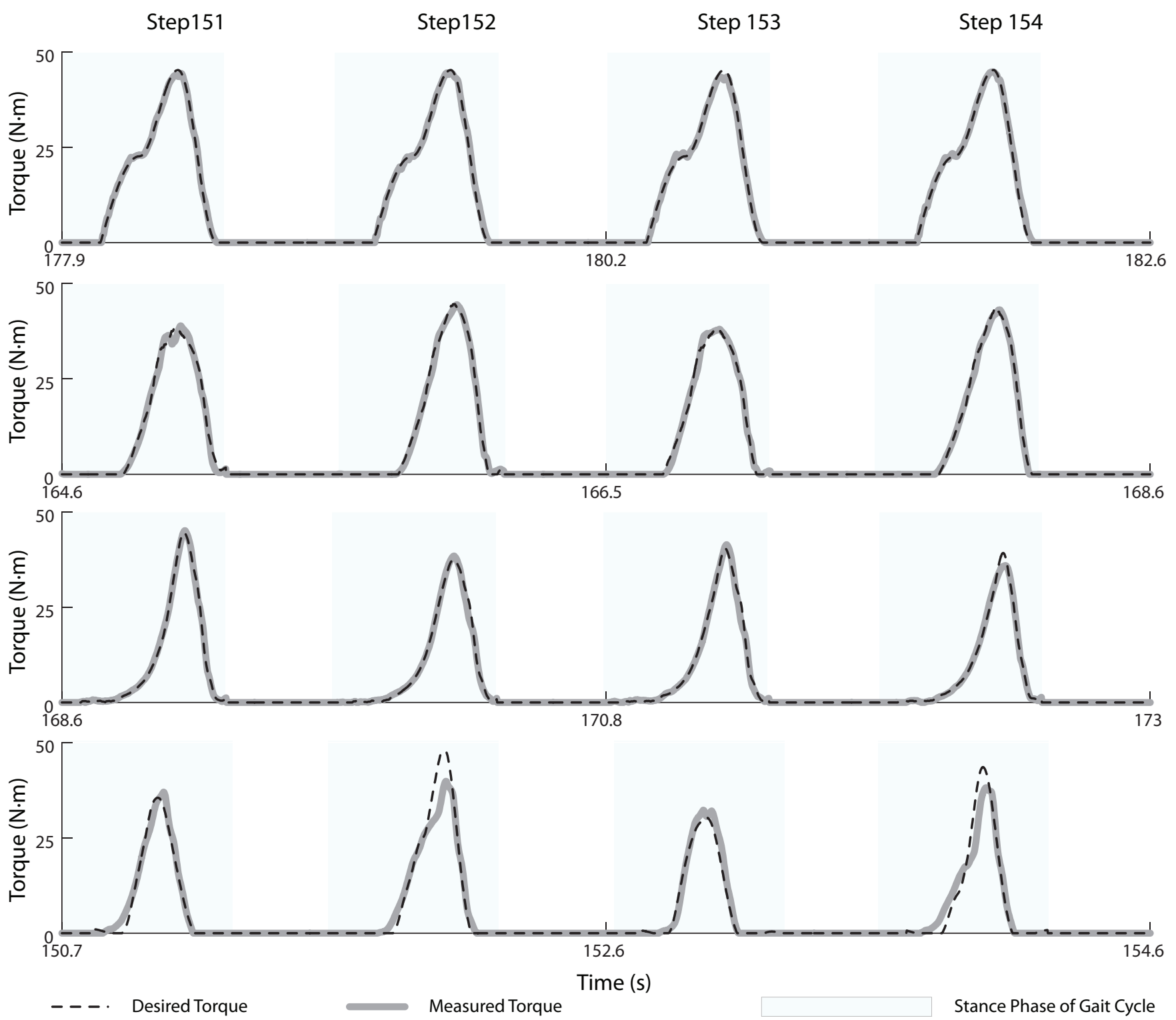

Fig. 8: Real-time Torque Tracking Performance of Proportional Control with Damping Injection Compensated by Iterative Learning. Trajectories of desired and measured torque for four consecutive steady state steps (Steps 151-154) were displayed for all four high-level controllers. Step-to-step variations of step duration and desired torque profile in Angle, NMM and EMG-based high level controllers are due to changes in human gait. 